\title{
Rousseau juge de Jean-Jacques. Etudes sur les Dialogues, sous la direction de Philip Knee et Gérard Allard
}

\section{Paola Sosso}

\section{(2) OpenEdition}

1 Journals

\section{Édition électronique}

URL : http://journals.openedition.org/studifrancesi/34763

DOI : 10.4000/studifrancesi.34763

ISSN : 2427-5856

Éditeur

Rosenberg \& Sellier

\section{Édition imprimée}

Date de publication : 1 novembre 2005

Pagination : 424

ISSN : 0039-2944

\section{Référence électronique}

Paola Sosso, «Rousseau juge de Jean-Jacques. Etudes sur les Dialogues, sous la direction de Philip Knee et Gérard Allard », Studi Francesi [En ligne], 146 (XLIX | II) | 2005, mis en ligne le 30 novembre 2015, consulté le 20 avril 2021. URL : http://journals.openedition.org/studifrancesi/34763 ; DOI : https:// doi.org/10.4000/studifrancesi.34763

Ce document a été généré automatiquement le 20 avril 2021.

\section{(c) $($ ) $\odot$}

Studi Francesi è distribuita con Licenza Creative Commons Attribuzione - Non commerciale - Non opere derivate 4.0 Internazionale. 


\title{
Rousseau juge de Jean-Jacques. Etudes sur les Dialogues, sous la direction de Philip Knee et Gérard Allard
}

\author{
Paola Sosso
}

\section{RÉFÉRENCE}

Rousseau juge de Jean-Jacques. Etudes sur les Dialogues, sous la direction de PHILIP KNEE et GÉRARD ALLARD, Paris, Champion, 2003, pp. 270.

1 Cet ouvrage est la réédition, revue et augmentée, du volume publié à l'origine dans la collection «Pensée libre $n^{\circ}$ 7» (Ottawa, 1998) par l'Association nord-américaine des études Jean-Jacques Rousseau. A une exception près, les textes ont été présentés lors d'un colloque bilingue tenu à l'Université Laval au Québec en 1997. L'introduction de Raymond TROUSSON (Rousseau et les Dialogues ou Jean-Jacques dans le labyrinthe, pp. 11-31) suit les étapes fondamentales du chemin qui a conduit Rousseau à substituer la peinture de son être authentique à celle qu'en proposent ses ennemis. On reparcourt toute une série de textes, en particulier la correspondance de 1770 (lettres à Mme de Berhtier, à Moultou, à $M$ de Saint Germain...), mais l'obsession du complot remonte plus loin, nous suggère Trousson, il suffit de penser à la condamnation de l'Emile et du Contrat Social. Les thèmes du labyrinthe et $d u$ double apparaissent continuellement pour représenter la vision erronée que les autres ont de Jean-Jacques, tandis que lui, de son côté, a une connaissance indiscutable de sa véritable identité, au point de transformer le genre du dialogue en une stratégie d'exposition. La première section («Monologue et dialogue») commence avec un article de Aubrey ROSEMBERG (Rousseau juge de Jean-Jacques: Dialogue ou Monologue?, pp. 35-44) où l'on montre qu'en réalité les Dialogues ne fournissent pas, comme le voudrait la tradition du genre, un authentique questionnement, parce que Rousseau est incapable de sortir de lui-même pour assumer un autre point de vue. L'étude de Judy GILLELAND s'interroge sur un thème semblable 
(Rousseau face à Jean-Jacques: la fonction et lafausseté du dialogue dans le premier Dialogue de Rousseau, pp. 45-56), tandis que Byron Wells (Language and Solitude: Paradox of the Dialogues, pp. 57-66) nous explique que les Dialogues, nés du silence qui suit les Confessions, ont leur origine dans la solitude et dans le désir de créer cet échange de points de vue que ses contemporains ne concedaient pas à Jean-Jacques. Rousseau and the Dialogue of Identity (pp. 67-78) de John T. scotT veut nous faire voir que les Dialogues ne sont pas un texte pathologique, mais se rattachent plutôt à une forme philosophique typique chez Rousseau, qui l'utilise à maintes reprises dans ses recherches sur l'homme et sur lui-même. La section se clôt avec un essai de Martine DROUET qui a pour titre Le jeu du dialogue dans les Dialogues (pp. 79-92).

2 «Le complot et la politique» est le titre de la deuxième section qui s'ouvre avec un article de Gérald ALLARD (Le Français juge de Jean-Jacques, pp. 95-106): l'auteur nous invite à une lecture dinamique du texte, semblable à celle que l'on fait d'habitude avec les œuvres de Platon, pour entrer dans la pensée des personnages et mieux cerner la position de Rousseau. Yves citTon dans son étude Fabrique de l'opinion et folie de la dissidence dans le complot selon Rousseau (107-122) nous propose de considérer le complot décrit par l'écrivain non pas comme une structure subjective, mais comme le symptôme d'une évolution socio-historique qui coïncide avec l'émergence de l'opinion publique. Ce point de vue nous aide à comprendre, selon Citton, que la vision délirante de Rousseau ne correspond pas à la réalité de son temps mais anticipe plutôt quelques éléments typiques de notre époque. Le texte qui suit, de Philip STEWART (Surveiller et entraver: les «gouverneurs» de Jean-Jacques; pp. 123-130), rapproche le couple Emile/ précepteur de celui des Dialogues, mettant en relation une série d'échos comparables et significatifs dans les deux œuvres. Le texte de Philip KNEE (L'amour propre et le complot dans les Dialogues, pp. 131-151) est suivi de la troisième section qui s'interroge sur «Le cœur et l'imagination».

3 Le premier essai de Michèle CROGIEZ (Le monstre et le mystère: le rôle de l'imagination dans les Dialogues de Rousseau, pp. 155-166) se concentre sur les différentes fonctions de l'imagination, tantôt positives, souvent négatives. L'imagination vive de l'écrivain se trouve face à l'imagination anarchique de ceux qui voient en lui un monstre. Sur un thème analogue, l'étude de Elaine LAROCHELLE (Jean-Jacques et ses chimères, pp. 167-180) nous présente une analyse du rapport troublant de Jean-Jacques avec le monde des chimères: l'importance accordée à l'imagination, selon Larochelle, est la cause première qui a conduit Rousseau à alimenter son cœur de fictions et d'êtres irréels. Michael o'DEA dans son article La voix et la fiction dans les Dialogues (pp. 181-194) part de la considération que le mot "cœur» est très utilisé dans les écrits de Rousseau dès les premiers textes: cette centralité du sentiment, symbole de la volonté de s'ouvrir à autrui, s'exprime souvent par la voix à l'intérieur de la fiction. C'est le cas par exemple du monde idéal du premier dialogue, qui expime cette conception de l'écrivain selon laquelle la parole sincère doit être imprégnée d'émotion. L'article de Pamela GAY-WHITE (Rousseau's Pygmalion: A Prelude to the Dialogues, pp. 195-203) rapproche les Dialogues d'un texte pas très lu, Pygmalion, pour un semblable désespoir et pour le fort désir de justification qui caractérise ces deux œuvres. En plus, la forme théâtrale sert dans les deux cas à révéler la pensée intime de l'auteur.

«L'homme et l'œuvre» est le titre de la dernière section, qui contient une première étude d'Ourida mostefai (Lecture d'une défiguration: la réception de l'auteur et de l'ouvre dans Rousseau juge de Jean-Jacques, pp. 207-216) dans laquelle on analyse le rapport 
difficile entre Rousseau et le Français, seul type de relation possible dans un monde où ne règne plus la transparence. Le texte de Laurence MALL, Mise en scène de «l'homme-etl'œuvre» dans les Dialogues (pp. 217-230) est suivi de celui de Gauthier AMBRUS, La figure $d u$ philosophe (pp. 231-242) où l'on fournit une analyse détaillée des rapports entre Rousseau, Diogène, Epictète et Socrate: à l'intérieur des Dialogues chacun de ces philosophes revêt une fonction spécifique, permettant à l'écrivain genevois de mieux définir son caractère à travers le recours à une série de références ayant chacune une signification bien claire aux yeux du lecteur. Le dernier texte de la section est de Guillemette JoHnston (Dire/médir Jean-Jacques ou lire/délire Rousseau: l'individuation dans les Dialogues, pp. 243-255): il nous offre une analyse du texte se basant sur le processus global d'individuation présenté en psychanalyse analytique. Le volume fournit encore l'Index des noms, l'Index des œuvres de Rousseau et une liste bibliographique. 